ventilation (to maintain $\mathrm{SpO}_{2} \geq 92 \%$ ) and Cohort 2 patients requiring mechanical ventilation, initiated $\leq 48$ hours before randomization. Here, we report results for Phase 2, Cohort 1: 116 patients with severe COVID- 19 pneumonia and hyperinflammation from USA, Brazil, Chile, Peru, and South Africa; randomized 1:1:1 to receive a single intravenous administration of mavrilimumab (10 or $6 \mathrm{mg} / \mathrm{kg}$ ) or placebo. The primary efficacy endpoint was proportion of patients alive and free of mechanical ventilation at Day 29. Secondary endpoints included [1] time to 2-point clinical improvement (National Institute of Allergy and Infectious Diseases COVID-19 ordinal scale), [2] time to return to room air, and [3] mortality, all measured through Day 29. The prespecified evidentiary standard was a 2 -sided $\alpha$ of 0.2 (not adjusted for multiplicity).

Results: Baseline demographics were balanced among the intervention groups; patients were racially diverse ( $43 \%$ non-white), had a mean age of 57 years, and $49 \%$ were obese $(\mathrm{BMI} \geq 30)$. All patients received the local standard of care: $96 \%$ received corticosteroids (including dexamethasone) and $29 \%$ received remdesivir. No differences in outcomes were observed between the $10 \mathrm{mg} / \mathrm{kg}$ and $6 \mathrm{mg} /$ $\mathrm{kg}$ mavrilimumab arms. Results for these groups are presented together. Mavrilimumab recipients had a reduced requirement for mechanical ventilation and improved survival: at day 29, the proportion of patients alive and free of mechanical ventilation was 12.3 percentage points higher with mavrilimumab $(86.7 \%$ of patients) than placebo $(74.4 \%$ of patients) (Primary endpoint; $p=0.1224)$. Mavrilimumab recipients experienced a $65 \%$ reduction in the risk of mechanical ventilation or death through Day 29 (Hazard Ratio $(H R)=0.35 ; p=0.0175$ ). Day 29 mortality was 12.5 percentage points lower in mavrilimumab recipients $(8 \%)$ compared to placebo $(20.5 \%)(p=0.0718)$. Mavrilimumab recipients had a $61 \%$ reduction in the risk of death through Day $29(\mathrm{HR}=0.39 ; \mathrm{p}=0.0726)$. Adverse events occurred less frequently in mavrilimumab recipients compared to placebo, including secondary infections and thrombotic events (known complications of COVID-19). Thrombotic events occurred only in the placebo arm (5/40 [12.5\%])

Mavrilimumab Reduced the Risk of Mechanical Ventilation or Death by $65 \%$ Versus Placebo

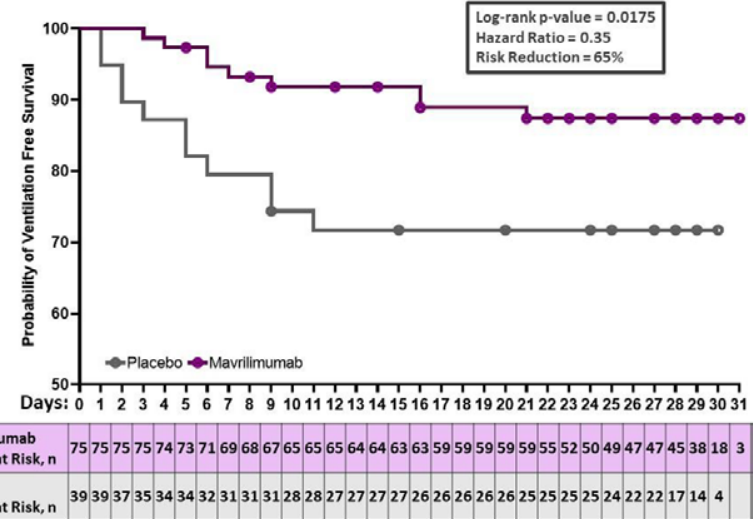

Conclusion: In a global, diverse population of patients with severe COVID-19 pneumonia and hyperinflammation receiving supplemental oxygen therapy, corticosteroids, and remdesivir, a single infusion of mavrilimumab reduced progression to mechanical ventilation and improved survival. Results indicate mavrilimumab, a potent inhibitor of GM-CSF signaling, may have added clinical benefit on top of the current standard therapy for COVID-19. Of potential importance is that this treatment strategy is mechanistically independent of the specific virus or viral variant. REFERENCES:

[1] Trapnell, Nat Rev Dis Pri, 2019

[2] Wicks, Nat Rev Immunology, 2015

[3] Hamilton, Exp Rev Clin Immunol, 2015

[4] De Luca, Lancet Rheumatol, 2020

[5] Cremer, Lancet Rheumatol, 2021

[6] Zhou, Nature, 2020

Disclosure of Interests: Lara Pupim Employee of: Kiniksa, Shareholder of: Kiniksa, Tisha S. Wang Consultant of: Partner Therapeutics; steering committee for Kinevant BREATHE clinical trial, Kristin Hudock: None declared, Joshua Denson: None declared, Nyda Fourie: None declared, Luis Hercilla Vasquez: None declared, Kleber Luz: None declared, Mohammad Madjid Grant/research support from: Kiniksa, Kirsten McHarry: None declared, José Francisco Saraiva: None declared, Eduardo Tobar: None declared, Teresa Zhou Employee of: Kiniksa, Shareholder of: Kiniksa, Manoj Samant Employee of: Kiniksa, Shareholder of: Kiniksa, Joseph Pirrello Employee of: Kiniksa, Shareholder of: Kiniksa, Fang Fang Employee of: Kiniksa, Shareholder of: Kiniksa, John F. Paolini Employee of: Kiniksa, Shareholder of: Kiniksa, Arian Pano Employee of: Kiniksa, Shareholder of: Kiniksa, Bruce C. Trapnell: None declared DOI: 10.1136/annrheumdis-2021-eular.5012

\section{LB0002 COVID-19 VACCINE SAFETY IN PATIENTS WITH RHEUMATIC AND MUSCULOSKELETAL DISEASE}

P. M. Machado ${ }^{1}$, S. Lawson-Tovey ${ }^{2}$, K. Hyrich ${ }^{2}$, L. Carmona ${ }^{3}$, L. Gossec ${ }^{4}$, E. Mateus ${ }^{5}$, A. Strangfeld ${ }^{6}$, B. Raffeiner ${ }^{7}$, T. Goulenok $^{8}$, O. Brocq $^{9}$, M. Cornalba ${ }^{10}$, J. A. Gómez-Puerta ${ }^{11}$, E. Veillard ${ }^{12}$, L. Trefond ${ }^{13}$, J. E. Gottenberg ${ }^{14}$, J. Henry ${ }^{15}$, P. Durez ${ }^{16}$, G. R. Burmester ${ }^{17}$, M. Mosca ${ }^{18}$ E. Hachulla ${ }^{19}$, H. Bijlsma ${ }^{20}$, I. McInnes ${ }^{21}$, X. Mariette ${ }^{22} .{ }^{1}$ University College London, Centre for Rheumatology and Department of Neuromuscular Diseases, London, United Kingdom; ${ }^{2}$ The University of Manchester, Centre for Epidemiology Versus Arthritis, Manchester, United Kingdom; ${ }^{3}$ Instituto de Salud Musculoesquelética, INMUSC, Madrid, Spain; ${ }^{4}$ Sorbonne Université, INSERM, Institut Pierre Louis d'Epidémiologie et de Santé Publique, Paris, France; ${ }^{5}$ Portuguese League Against Rheumatic Diseases, LPCDR, Lisbon, Portugal; ${ }^{6}$ German Rheumatism Research Center (DRFZ Berlin), Epidemiology and Health Care Research, Berlin, Germany; ${ }^{7}$ Central Hospital of Bolzano, Rheumatology, Bolzano, Italy; ${ }^{8}$ Bichat -Claude Bernard Hospital, APHP, Department of Internal Medicine, Paris, France; ${ }^{9} \mathrm{CHPG}$, Department of Rheumatology, Monaco, Monaco; ${ }^{10}$ ASST Gaetano Pini - CTO Institute, Division of Clinical Rheumatology, Milan, Italy; ${ }^{11}$ Hospital Clinic, Department of Rheumatology, Barcelona, Spain; ${ }^{12}$ Cabinet de Rhumatologie des "Marines de Chasles", Department of Rheumatology, Saint Malo, France; ${ }^{13} \mathrm{CHU}$ GABRIEL Montpied, Department of Rheumatology, Clermont Ferrand, France; ${ }^{14}$ Hopital de Hautepierre, Department of Rheumatology, Strasbourg, France; ${ }^{15}$ Hopitaux Universitaires Paris Sud, Department of Rheumatology, Paris, France; ${ }^{16}$ Cliniques Universitaires Saint-Luc, Department of Rheumatology, Brussels, Belgium; ${ }^{17}$ Charité - Universitätsmedizin, Department of Rheumatology and Clinical Immunology, Berlin, Germany; ${ }^{18}$ University of Pisa, Department of Rheumatology, Pisa, Italy; ${ }^{19}$ Lille University, Hopital Claude Huriez, Lille, France; ${ }^{20}$ University of Utrecht, Department of Rheumatology, Utrecht, Netherlands; ${ }^{21}$ University of Glasgow, Institute for Infection, Immunity and Inflammation, Glasgow, United Kingdom; ${ }^{22}$ Hôpital Bic être, Assistance Publique-Hôpitaux de Paris, Department of Rheumatology, Paris, France

Background: The consequences of the COVID-19 outbreak are unprecedented and have been felt by everyone around the world, including people with rheumatic and musculoskeletal diseases (RMDs). With the development of vaccines, the future is becoming brighter. Vaccines are a key pillar of public health and have been proven to prevent many serious diseases. However, vaccination also raises questions, especially for patients with inflammatory RMDs and/or treated with drugs that influence their immune system.

Objectives: Our aim was to collect safety data among RMD patients receiving COVID-19 vaccines.

Methods: The EULAR COVID-19 Vaccination (COVAX) Registry is an observational registry launched on 5 February 2021. Data are entered voluntarily by clinicians or associated healthcare staff; patients are eligible for inclusion if they have an RMD and have been vaccinated against SARS-CoV-2. Descriptive statistics are presented. Results: As of 27 April 2021, 1519 patients were reported to the registry. The majority were female $(68 \%)$ and above the age of $60(57 \%)$. Mean age was 63 years (SD 16), ranging from 15 to 97 years. A total of 28 countries contributed to the registry, with France $(60 \%)$ and Italy $(13 \%)$ as the highest contributors. The majority $(91 \%)$ had inflammatory RMDs. Inflammatory joint diseases accounted for $51 \%$ of cases, connective tissue diseases $19 \%$, vasculitis $16 \%$, other immune mediated inflammatory diseases $4 \%$, and non-inflammatory/mechanical RMDs $9 \%$. The most frequent individual diagnoses were rheumatoid arthritis $(30 \%)$, axial spondyloarthritis $(8 \%)$, psoriatic arthritis (8\%), systemic lupus erythematosus (SLE, 7\%) and polymyalgia rheumatica ( $6 \%$ ). At the time of vaccination, $45 \%$ were taking conventional synthetic DMARDs, $36 \%$ biological DMARDs, $31 \%$ systemic glucocorticoids, $6 \%$ other immunosuppressants (azathioprine; mycophenolate; cyclosporine; cyclophosphamide; tacrolimus), and $3 \%$ targeted synthetic DMARDs. The most frequent individual DMARDs were methotrexate (29\%), TNF-inhibitors (18\%), antimalarials (10\%) and rituximab (6\%). The vaccines administered were: $78 \%$ Pfizer, $16 \%$ AstraZeneca, $5 \%$ Moderna and $1 \%$ other/unknown; $66 \%$ of cases received two doses and $34 \%$ one dose. Mean time from 1 st and 2 nd dose to case report was 41 days (SD 26) and 26 days (SD 23), respectively. COVID-19 diagnosis after vaccination was reported in $1 \%(18 / 1519)$ of cases. Mean time from first vaccination until COVID-19 diagnosis was 24 days (SD 17). Disease flares were reported by $5 \%(73 / 1375)$ of patients with inflammatory RMDs, with $1.2 \%$ (17/1375) classified as severe flares. Mean time from closest vaccination date to inflammatory RMD flare was 5 days (SD 5). The most common flare types were arthritis $(35 / 1375=2.5 \%)$, arthralgia $(29 / 1375=2.1 \%)$, cutaneous flare $(11 / 1375=0.8 \%)$ and increase in fatigue $(11 / 1375=0.8 \%)$. Potential vaccine side effects were reported by $31 \%$ of patients (467/1519). The majority were typical early adverse events within 7 days of vaccination, namely pain at the site of injection (281/1519=19\%), fatigue (171/1519=11\%) and headache (103/1519=7\%). Organ/system adverse events were reported by $2 \%$ (33/1519) but only $0.1 \%$ (2/1519) reported 
severe adverse events, namely a case of hemiparesis in a patient with systemic sclerosis/SLE overlap syndrome (ongoing at the time of reporting), and a case of giant cell arteritis in a patient with osteoarthritis (recovered/resolved without sequelae). Conclusion: The safety profiles for COVID-19 vaccines in RMD patients was reassuring. Most adverse events were the same as in the general population, they were non-serious and involved short term local and systemic symptoms. The overwhelming majority of patients tolerated their vaccination well with rare reports of inflammatory RMD flare (5\%; $1.2 \%$ severe) and very rare reports of severe adverse events $(0.1 \%)$. These initial findings should provide reassurance to rheumatologists and vaccine recipients, and promote confidence in COVID-19 vaccine safety in RMD patients, namely those with inflammatory RMDs and/or taking treatments that influence their immune system.

Acknowledgements: EULAR COVID-19 Task Force; European Reference Network on rare and Complex Connective Tissue and Musculoskeletal Diseases; European Reference Network on Rare Immunodeficiency, Autoinflammatory and Autoimmune Diseases Network; all rheumatologists contributing to the EULAR COVAX Registry.

Disclosure of Interests: Pedro M Machado Consultant of: Abbvie, BMS, Celgene, Eli Lilly, Janssen, MSD, Novartis, Orphazyme, Pfizer, Roche and UCB, all unrelated to this manuscript., Grant/research support from: Orphazyme, unrelated to this manuscript., Speakers bureau: Abbvie, BMS, Celgene, Eli Lilly, Janssen, MSD, Novartis, Orphazyme, Pfizer, Roche and UCB, all unrelated to this manuscript., Saskia Lawson-Tovey: None declared, Kimme Hyrich Grant/research support from: BMS, UCB, and Pfizer, all unrelated to this manuscript., Speakers bureau: Abbvie, Loreto Carmona Consultant of: her institute works by contract for laboratories among other institutions, such as Abbvie Spain, Eisai, Gebro Pharma, Merck Sharp \& Dohme España, S.A., Novartis Farmaceutica, Pfizer, Roche Farma, Sanofi Aventis, Astellas Pharma, Actelion Pharmaceuticals España, Grünenthal GmbH, and UCB Pharma, all unrelated to this manuscript., Laure Gossec Grant/research support from: AbbVie, Amgen, BMS, Biogen, Celgene, Gilead, Janssen, Lilly, Novartis, Pfizer, Samsung Bioepis, Sanofi-Aventis, UCB, all unrelated to this manuscript., Speakers bureau: Amgen, Lilly, Janssen, Pfizer, Sandoz, Sanofi, Galapagos, all unrelated to this manuscript., Elsa Mateus Grant/research support from: LPCDR received support for specific activities: grants from Abbvie, Novartis, Janssen-Cilag, Lilly Portugal, Sanofi, Grünenthal S.A., MSD, Celgene, Medac, Pharmakern, GAfPA; grants and non-financial support from Pfizer; non-financial support from Grünenthal $\mathrm{GmbH}$, outside the submitted work., Anja Strangfeld Speakers bureau: AbbVie, MSD, Roche, BMS, and Pfizer, all unrelated with this manuscript., BERND RAFFEINER: None declared, Tiphaine Goulenok: None declared, Olilvier Brocq: None declared, Martina Cornalba: None declared, José A Gómez-Puerta Speakers bureau: AbbVie, BMS, GSK, Janssen, Lilly, MSD, Roche and Sanofi., Eric Veillard: None declared, Ludovic Trefond: None declared, Jacques-Eric Gottenberg: None declared, Julien Henry: None declared, Patrick Durez: None declared, Gerd Rüdiger Burmester: None declared, Marta Mosca: None declared, Eric Hachulla: None declared, Hans Bijlsma: None declared, lain Mclnnes: None declared, Xavier Mariette Consultant of: BMS, Galapagos, Gilead, Janssen, Novartis, Pfizer, Sanofi-Aventis, UCB, and grant from Ose, all unrelated to this manuscript.

DOI: 10.1136/annrheumdis-2021-eular.5097

\section{LB0003 \\ IMMUNOGENICITY AND SAFETY OF THE BNT162B2 MRNA COVID-19 VACCINE IN ADULT PATIENTS WITH AUTOIMMUNE INFLAMMATORY RHEUMATIC DISEASES AND GENERAL POPULATION: A MULTICENTER STUDY}

$\underline{\text { V. Furer }}^{1}$, T. Eviatar ${ }^{1}$, D. Zisman², H. Peleg ${ }^{3}$, D. Paran ${ }^{1}$, D. Levartovsky ${ }^{1}$, M. Zisapel ${ }^{1}$, O. Elalouf ${ }^{1}$, I. Kaufman ${ }^{1}$, R. Meidan ${ }^{1}$, A. Broyde ${ }^{1}$, A. Polachek ${ }^{1}$, J. Wollman ${ }^{1}$, I. Litinsky ${ }^{1}$, K. Meridor ${ }^{1}$, H. Nochomovitz ${ }^{1}$, A. Silberman ${ }^{1}$, D. Rosenberg ${ }^{1}$, J. Feld ${ }^{2}$, A. Haddad ${ }^{2}$, T. Gazitt ${ }^{2}$, M. Elias ${ }^{2}$, N. Higazi ${ }^{2}$, F. Kharouf ${ }^{3}$, G. Shefer ${ }^{4}$, O. Sharon ${ }^{4}$, S. Pel ${ }^{1}$, S. Nevo ${ }^{1}$, O. Elkayam ${ }^{1} .{ }^{1} \mathrm{Tel}$ Aviv Medical Center, Rheumatology, Tel Aviv, Israel; ${ }^{2}$ Carmel Medical Center, Rheumatology, Haifa, Israel; ${ }^{3} \mathrm{Hadassah}$ Medical Center, Rheumatology, Jerusalem, Israel; ${ }^{4}$ Tel Aviv Medical Center, Endocrinology, Tel Aviv, Israel

Background: Vaccination represents a cornerstone in mastering the COVID-19 pandemic. Data on immunogenicity, efficacy, and safety of the novel BNT162b2 mRNA vaccine in patients with autoimmune inflammatory rheumatic diseases (AIIRD) are limited.
Objectives: To investigate the immunogenicity, efficacy, and safety of the BNT162b2 mRNA vaccine in patients with AlIRD compared to the general population.

Methods: A prospective multicenter study investigated immunogenicity, efficacy, and safety of the two-dose regimen BNT162b2 mRNA vaccine in adult patients with AIIRD including rheumatoid arthritis (RA), psoriatic arthritis (PsA), axial spondyloarthropathy ( $\operatorname{axSpA})$, systemic lupus erythematosus (SLE), connective tissues diseases (CTD), systemic vasculitides, and idiopathic inflammatory myositis (IIM), compared to control subjects without rheumatic diseases or immunosuppressive therapies. Serum IgG antibody levels against SARS-CoV-2 spike S1/S2 proteins were measured 2 - 6 weeks after the second vaccine dose. Seropositivity was defined as $\lg \mathrm{G} \geq 15$ binding antibody units $(B A U) / \mathrm{ml}$. Post-vaccination efficacy defined as post-vaccination COVID-19 infection and safety were assessed. Preand post- vaccination disease activity indices were assessed as appropriate for each disease.

Results: A total of 686 AlIRD patients and 121 controls participated into the study. AIIRD patients were significantly older than controls, mean age \pm SD $56.76 \pm 14.88$ vs $50.76 \pm 14.68$, respectively, $p<0.0001$. A total of $95.2 \% \quad(n=653)$ AlIRD patients were treated with immunomodulatory medications.

The seropositivity rate was $86 \%(n=590)$ in patients with AlIRD compared to $100 \%$ in controls $(p<0.0001$ ) The level of the S1/S2 antibodies was significantly reduced in AlIRD patients compared to controls (mean \pm SD $132.9 \pm 91.7$ vs 218.6 $\pm 82.06, P<0.0001)$. In patients with $P s A, A x S p A, S L E$, and LVV, the seropositive rate was above $90 \%$. In RA, the seropositive rate was $82.1 \%$ and the lowest seropositive rate $(<40 \%)$ was observed in patients with AAV and IIM. Anti-CD20 significantly impaired the vaccine's immunogenicity, with the lowest seropositivity rate of $39 \%$. The use of GC, mycophenolate mofetil (MMF), and abatacept was associated with a significantly lower rate of seropositivity (Figure 1). MTX significantly reduced the seropositivity in patients treated with MTX monotherapy and in combinations with other treatments $(92 \%$ and $84 \%$, respectively), although at a lesser magnitude than anti-CD20, MMF, and abatacept. More than $97 \%$ of patients treated with anti-cytokine therapies including TNFi, interleukin-17 and interleukin- 6 inhibitors had an appropriate immunogenic response when used as monotherapy. The combination of TNFi with MTX significantly reduced the rate of seropositivity to $93 \%, p=0.04$. Age over 65 years, a diagnosis of RA, IIM, ANCA-associated vascilitis, and treatment with GC, MMF, anti-CD20, and abatacept were associated with a reduced likelihood of seropositivity.

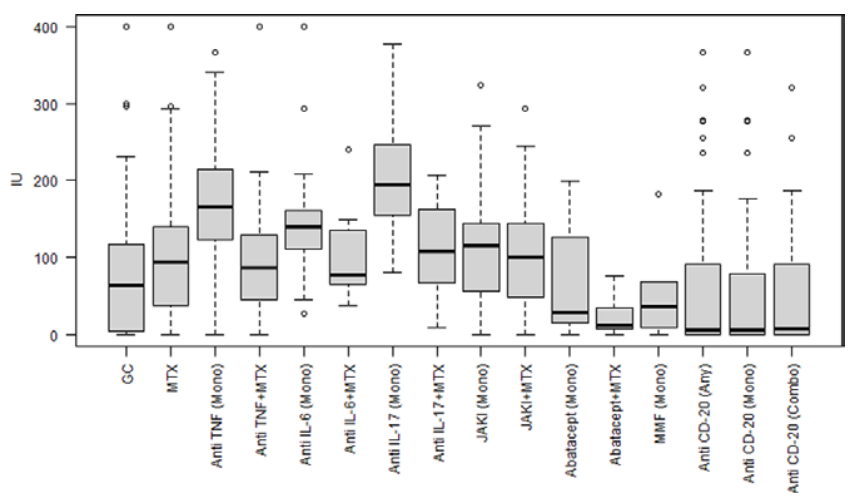

Figure 1. Seropositivity rate by immunosuppressive treatment.

There were no post-vaccination symptomatic cases of COVID-19 among AIIRD patients and one mild case in the control group. Major adverse events in AIIRD patients included death $(n=2)$ several weeks after the second vaccine dose, non-disseminated herpes zoster $(n=6)$, uveitis $(n=2)$, and pericarditis $(n=1)$. Post-vaccination disease activity remained stable in the majority of patients.

Conclusion: Vaccination with the BNTb262 vaccine resulted in an adequate immunogenic response with an acceptable safety profile in the majority of patients with AIIRD. Treatment with GC, rituximab, MMF, and abatacept may impair BNT162b2-induced immunogenicity. Postponing administration 